\title{
La place des Maronites à Livourne
}

\section{Guido Bellatti Ceccoli}

\section{(2) OpenEdition}

Journals

Édition électronique

URL : http://journals.openedition.org/edl/484

DOI : $10.4000 /$ edl. 484

ISSN : 2296-5084

\section{Éditeur}

Université de Lausanne

\section{Édition imprimée}

Date de publication : 15 mai 2013

Pagination : 75-90

ISBN : 978-2-940331-32-1

ISSN : 0014-2026

\section{Référence électronique}

Guido Bellatti Ceccoli, «La place des Maronites à Livourne », Études de lettres [En ligne], 1-2 | 2013, mis en ligne le 15 mai 2016, consulté le 22 décembre 2020. URL : http://journals.openedition.org/edl/484 ; DOI : https://doi.org/10.4000/edl.484 


\section{LA PLACE DES MARONITES À LIVOURNE ${ }^{1}$}

L'Eglise maronite, catholique de rite oriental, est née au IV e siècle et s'est développée dans la région syro-libanaise. Beaucoup de Maronites, depuis longtemps arabophones, ont migré à l'étranger pour des raisons commerciales. Certains se sont notamment installés à Livourne, ville marchande. Grâce aux recherches effectuées par l'auteur, on sait que la présence des Maronites dans le port toscan est attestée dès 1734. Des tombes révèlent la présence et le statut des Maronites à Livourne. Les épitaphes mortuaires sont écrites en arabe et en latin ou en arabe et en italien. Dans cet article sont publiées les traductions inédites (en français) de ces textes arabes, poèmes d'une valeur historique et culturelle indiscutable.

\section{Livourne, ville multiculturelle}

L'épanouissement de la ville de Livourne date du XVI e siècle. Considérée jusqu’à la fin du Moyen Age comme une dépendance du port de Pise, grande république maritime et antagoniste de la puissance vénitienne en Méditerranée, Livourne devient, à partir du XVI e siècle et grâce aux Médicis, le port principal du grand-duché de Toscane, état qui ne cache pas ses ambitions de devenir une grande puissance marchande et militaire en Méditerranée. Les Médicis «refondent» Livourne dans

I. Ce travail est la version révisée du texte de mon intervention au colloque «La représentation de l'espace dans les sciences humaines", organisé à l'Université de Lausanne les 12 et 13 mai 2011. Je saisis l'occasion pour remercier chaleureusement Monsieur Alberto Roncaccia, organisateur du colloque, de m'avoir permis d'y participer. 
cette optique, et ils invitent les marchands du monde entier à s'y établir, en particulier grâce aux leggi liburnine promulguées par le grand-duc Ferdinand I ${ }^{\text {er }}$ en 1591 et 1593 . Ces lois font expressément référence aux "Levantini, Ponentini, Spagnoli, Portoghesi, Greci, Tedeschi, Italiani, Ebrei, Turchi, Mori, Armeni, Persiani, ed altri ». Ainsi, au cours des années, le port devient une mosaïque de peuples, de cultures, de langues et de religions différents, qui cohabitent paisiblement avec l'objectif de s'enrichir. La population se compose de Juifs, d'Arméniens, d'Allemands, de Hollandais, d'Anglais, d'Ecossais, de Danois, de Syriens, de Libanais, d'Egyptiens, de Grecs, de Corses et de bien d'autres encore. Il y a également de nombreux musulmans, mais la plupart d'entre eux sont réduits en esclavage, proies des corsaires toscans, et notamment des chevaliers de Saint-Etienne. La présence de tous ces étrangers engendre, entre le XVI ${ }^{\mathrm{e}}$ et le XIX siècle, la naissance de communautés religieuses très variées qui disposent souvent d'un lieu de culte, parfois imposant. Au début du $\mathrm{XVII}^{\mathrm{e}}$ siècle, par exemple, il semble que la synagogue de Livourne ait été la plus grande d'Europe après celle d'Amsterdam. Les chrétiens non catholiques étaient nombreux: luthériens, calvinistes, vaudois, anglicans, presbytériens écossais, orthodoxes. Les catholiques étaient également divisés en groupes, dont la plupart étaient évidemment des Latins (les Livournais autochtones en premier lieu). On notera aussi l'existence de fidèles de certaines Eglises orientales rattachées à Rome: l'Eglise grecque "unie», dont faisaient partie des arabophones, les Melkites, qui étaient originaires d'anciens territoires byzantins arabisés à partir du VII ${ }^{\mathrm{e}}$ siècle, et l'Eglise maronite, composée surtout de Libanais, de Syriens et de Palestiniens, tous arabophones. Il y avait également de nombreux arabophones dans la communauté arménienne, car ses fidèles étaient souvent originaires de la région libanaise.

Livourne, port franc, a donné à tous ces immigrés des avantages fiscaux et un statut privilégié qui ont fait la fortune du commerce sur mer, surtout avec l'Orient, jusqu'à l'arrivé des Savoie. En effet, suite à l'unité italienne (1861), le port franc sera aboli et la ville perdra une grande partie de son intérêt aux yeux des marchands internationaux. Cela a causé le départ de nombreux Maronites; leur communauté a presque disparu avant la Deuxième Guerre mondiale. Au-delà de leur importance en tant que marchands, ils ont laissé une trace remarquable sur le plan culturel et historique. En effet, il ne faut pas oublier que le développement économique ne doit pas être surévalué au détriment des aspects culturels, 
y compris religieux et linguistiques, qui méritent encore des approfondissements. Dans ce contexte plus général, il convient de prendre en compte les mots du Professeur Riccardo Burigana ${ }^{2}$, spécialiste d'histoire religieuse, qui a affirmé récemment que "la ville de Livourne a un port et un chantier [naval], mais [qu'] elle n'est pas un port et un chantier». Elle est en effet beaucoup plus que cela.

\section{L'Eglise maronite}

L'Eglise maronite, fondée par Marûn, moine du IV siècle (350-433), s'est développée principalement au $\mathrm{V}^{\mathrm{e}}$ et au $\mathrm{VI}^{\mathrm{e}}$ siècles au Liban. Les disciples de Marûn ont bâti un monastère à Hamah, sur le fleuve Oronte. La liturgie maronite est syriaque (rite antiochien), avec des influences orientales et latines. La liturgie de l'eucharistie est une variante de celle de Saint-Jacques. La langue liturgique est l'arabe (c'était à l'origine le syriaque, jusqu'à l'arabisation de la région au VII siècle). Les Maronites se considèrent liés depuis toujours à l'autorité religieuse du pape de Rome; ils sont donc catholiques ${ }^{3}$. Des communautés maronites établies depuis longtemps existent également en dehors du Liban, dans les pays qui l'entourent, surtout en Syrie, mais aussi dans plusieurs pays de la Méditerranée comme la France et l'Italie, essentiellement pour des raisons commerciales ${ }^{4}$.

2. Monsieur Burigana a écrit de nombreuses publications sur l'histoire de la Toscane et de Livourne en portant une attention particulière à l'histoire des milieux ecclésiastiques. A Livourne, il a dirigé un centre sur l'œcuménisme qui a permis d'étudier, tant qu'il était sous sa direction, une nouvelle manière de concevoir l'histoire des villes dans un contexte global, en reconnaissant à l'élément religieux sa juste valeur. Actuellement, il dirige un centre d'études à Venise, auprès de l'ISE (Institut d'études œcuméniques San Bernardino), qui fait partie de l'Université pontificale Antonianum.

3. G. Bellatti Ceccoli, Tra Toscana e Medioriente, p. 45-51.

4. Sur la riche historiographie existant à propos de la communauté maronite, voir G. Bellatti Ceccoli, Tra Toscana e Medioriente, p. 361-378; voir également M. Moosa, The Maronites in the History, qui traite notamment de la "délicate question" (touchy question) du monothélisme. 


\section{Les Maronites à Livourne}

Pendant longtemps, on a affirmé que les premiers Maronites installés à Livourne étaient arrivés dans le port comme accompagnateurs de l'émir druze libanais Fakhr ad-Dîn en 1613. L'émir cherchait à obtenir l'aide diplomatique et militaire des Médicis, du pape et des Espagnols pour créer un état libanais affranchi de l'autorité ottomane. Dans un premier temps, j’ai suivi cette théorie, présentée comme une évidence dans de nombreux textes d'histoire livournaise, mais j'ai ensuite dû la remettre en question, car il n'existe aucune preuve d'une présence maronite stable à Livourne dès $1613^{5}$. En effet, cette présence est attestée avec certitude seulement à partir de 1734, vers la fin de la dynastie des Médicis (qui trouve son aboutissement en 1737 avec le décès du grand-duc Gian Gastone), quand le premier religieux maronite libanais s'est installé dans la ville pour confesser les autres arabophones (résidents ou de passage dans le port) et pour convertir au catholicisme les "infidèles» (et en particulier les esclaves musulmans, encore nombreux dans les bagnes de Livourne). Il a été nommé "Confesseur des Arabes» en 1736, et l'année suivante le premier grand-duc lorrain a confirmé sa nomination pour un temps indéterminé. De plus, lui et les confesseurs qui lui ont succédé (tous Maronites) ont toujours assuré, jusqu'en 1939, la gestion d'une institution religieuse "arabe» (la Cappellania Niccola Frangi), créée en 1755 par testament par un marchand melkite originaire de Damas pour garantir l'assistance spirituelle à tous les arabophones catholiques ${ }^{6}$.

$\mathrm{Au} \mathrm{XIX}^{\mathrm{e}}$ siècle, certains Maronites originaires d'Alep avaient une place socialement très importante à Livourne. Dans cette partie de la Toscane centrée sur le commerce maritime avec l'Orient, deux familles syriennes ont laissé une trace remarquable: les Cubbe (du nom arabe Kûbbâ) et les D'Andrea (probablement du nom arabe Andréaus). La présence de certains membres illustres de ces familles est attestée, entre autres, par quatre épitaphes funéraires qui se trouvent dans deux lieux de culte bien connus: le cloître de l'église de Saint-François (en plein

5. G. Bellatti Ceccoli, Tra Toscana e Medioriente, p. 58-61.

6. Les recteurs de la Cappellania Niccola Frangi étaient nommés par la congrégation vaticane de Propaganda fide. Sur cette Cappellania ont été redigés deux mémoires de maîtrise, malheureusement jamais publiés, par Monsieur Gianluca Pellegrini (1990) et par Madame Lorenza Litrico (1999), tous deux sous la direction du Professeur Cesare Alzati de l'Université de Pise (Faculté de Lettres et Philosophie). 
centre ville de Livourne) et le Sanctuaire de la Vierge de Montenero (voir ci-dessous la partie "Epitaphes maronites à Livourne»). Ces pierres tombales sont toutes bien visibles aux visiteurs, car elles sont disposées verticalement, à hauteur d'homme, à coté de l'entrée principale du sanctuaire (sous le portique) et dans le cloître des Franciscains.

Pour comprendre les liens forts qui se sont créés entre la communauté latine «autochtone» et la communauté «immigrée» des catholiques arabophones, il est important de signaler qu'entre 1834 et 1840, l'évêque de Livourne était un Maronite: Raphaël Cubbe, né en 1772 à Alep et arrivé en Toscane quand il était encore un enfant, entre 1776 et $1778^{7}$.

Pour recueillir les confessions des fidèles arabophones (Melkites, Maronites ou d'autres groupes), les moines maronites ont bénéficié de l'hospitalité de l'église dominicaine de Sainte-Catherine (de rite latin), dans l'ancienne chapelle de Saint-Thomas. Même s'ils n'ont jamais été très nombreux, les Maronites ont toujours voulu disposer d'un propre lieu de culte où célébrer leurs rites, et en particulier la messe en arabe (avec la liturgie antiochienne). Mais il n'a pas été facile de l'obtenir. Ce n'est qu'en 1889, et après avoir rejeté de nombreuses requêtes, que l'évêque de Livourne, Leopoldo Franchi, a autorisé l'ouverture d'un lieu de prière (rien de plus). Et il faudra attendre 1900 pour que soit érigée, dans le même édifice, la première (et dernière) paroisse maronite de Livourne. Il s'agissait d'une paroisse "nationale" sans circonscription territoriale définie, à savoir ouverte à tous les membres de la «nation» maronite, résidents ou de passage (mais l'église était ouverte également aux catholiques latins, évidemment). L'église, appelée «chiesa della Santissima Vergine del Rosario della Valle di Pompei ", à l'origine de modestes dimensions, a été ensuite élargie et, en 1921, a officiellement été déclarée «Sanctuaire». Pourtant, les fidèles maronites étaient de moins en moins nombreux, et le quartier où se trouvait le lieu de culte devenait de plus en plus peuplé. Ainsi, des fidèles avaient demandé, en 1923, s'ils pouvaient assister à la messe "chez les Maronites", car leurs paroisses latines respectives étaient plus éloignées de leurs domiciles. A commencé alors à se former, dans le clergé local, l'idée de transformer ce lieu de culte en église de rite latin, avec une circonscription territoriale qui aurait absorbé une partie des circonscriptions des paroisses

7. Sur la vie et l'œuvre de Mgr. Raphaël Cubbe, je renvoie à G. Bellatti Ceccoli, «Da Kûbbâ a Livorno ». 
environnantes (Santa Maria del Soccorso, SS. Trinità, San Jacopo et Ardenza), allégeant ainsi la charge de travail de leurs curés. Il y avait toutefois un obstacle de taille: l'édifice de l'église maronite avait été offert par des particuliers (les frères Borghini), et ensuite légué par testament à l'Eglise par le père Notain Darauni, mais à condition qu'il restât à jamais un lieu de culte de rite maronite. L'abandon d'une telle clause nécessitait l'intervention du pape en personne, le seul ayant l'autorité pour "modifier la volonté des donateurs et du testateur" afin de permettre le passage de la paroisse au rite latin, passage qui est intervenu en 1938. L'année suivante l'évêque de Livourne a donc pu procéder, par décret, à la création de la circonscription de la paroisse "ex-maronite» et, en même temps, à la redéfinition des circonscriptions paroissiales voisines.

L'église maronite avait une architecture sobre et élégante qui remontait au XVIII e siècle. Toutefois, ce beau sanctuaire, malgré son élégance et son passé glorieux, et malgré la chance qu'il avait eue de ne pas être détruit par les bombardements, a été complètement rasé après la Deuxième Guerre mondiale. A sa place a été bâtie une nouvelle église, plus spacieuse, certes, mais d'une valeur esthétique très discutable. Elle a rouvert ses portes aux fidèles en 1963 seulement, après un quart de siècle de fermeture.

Il n'existe que peu de publications spécifiques sur les Maronites à Livourne, car leur présence n'a été étudiée en profondeur que récemment ${ }^{8}$. Parmi les rares œuvres du passé, il convient de signaler, surtout pour les documents cités ou transcrits, celles de deux curés arabes qui ont marqué l'histoire de cette communauté religieuse à Livourne entre 1885 et la veille de la Deuxième Guerre mondiale?

8. Voir en particulier G. Bellatti Ceccoli, "Voci dall'Oriente»; Tra Toscana e Medioriente, p. 45-51 et 55-220 ; "Medioriente livornese»; «Da Kûbbâ a Livorno»; et avec G. Di Nicola: "Segni orientali nella Livorno ottocentesca» et «Lapidi arabe a Livorno». Voir également l'étude de L. Litrico, "Un cedro del Libano in Toscana».

9. G. Notain Darauni, Cenni storici sulla nazione siro-maronita; T. Anaissi, Collectio Documentorum Maronitarum. 
Epitaphes maronites à Livourne

\section{Prénom, nom, dates de} naissance et de décès

1. Nàamat-Allah Cubbe (Alep 1752 - Livourne 1828)

2. Michel Cubbe (Alep 1761 - Livourne 1830)

3. Jean D'Andrea (Alep 1770 - Casciana 1839)

4. Louis Cubbe (Alep 1763 - Livourne 1842)
Langues Lieu de sépulture

$\begin{array}{cl}\text { latin et arabe } & \begin{array}{l}\text { Eglise de Saint-François, } \\ \text { Livourne }\end{array} \\ \text { latin et arabe } & \begin{array}{l}\text { Sanctuaire de la Vierge } \\ \text { de Montenero }\end{array} \\ \text { italien et arabe } & \begin{array}{l}\text { Sanctuaire de la Vierge } \\ \text { de Montenero }\end{array} \\ \text { latin et arabe } & \begin{array}{l}\text { Sanctuaire de la Vierge } \\ \text { de Montenero }\end{array}\end{array}$

Les textes sculptés sur ces tombes montrent clairement le rapport complexe entretenu par les Maronites avec leur patrie d'origine et leur lieu d'adoption, relation oscillant entre une synthèse et une dualité identitaire, qui a marqué plusieurs générations de ces «chrétiens orientaux». Cela s'est produit au cours d'un processus d'assimilation achevé au $\mathrm{XX}^{\mathrm{e}}$ siècle, lorsque tous ceux qui n'ont pas quitté Livourne se sont complètement «latinisés». La dimension linguistique joue un rôle essentiel dans cette "identité multiple», mais sans se figer: au fil du temps, ces Maronites, dans le contexte italien et sans amoindrir l'importance de l'arabe originaire, ont reconnu une place de premier plan à l'italien, langue d'usage quotidien, mais également au latin, langue officielle de l'Eglise catholique, qui a toujours constitué, pour eux, un repère identitaire fort.

Je présente ici les textes en latin et le texte en italien, suivis par les traductions en français des textes arabes. Pour les textes en arabe, je renvoie à une publication en italien ${ }^{10}$ agrémentée de plusieurs illustrations en

Io. G. Bellatti Ceccoli, G. Di Nicola, «Segni orientali nella Livorno ottocentesca». Cette publication n'est pas facile à trouver, mais elle peut être demandée à l'adresse e-mail cangido@hotmail.com. Je signale également la publication du même article (en italien) sur le site internet brésilien www.cehilanet.net. 
couleur. Il s'agit ici de la première publication de ces traductions en français ${ }^{11}$.

La différence fondamentale entre les textes en latin et italien et les textes en arabe est que les premiers sont plus descriptifs, presque administratifs (faisant référence aux dates et aux lieux de naissance et de mort, à la ville d'origine, aux titres de noblesse et aux membres de la famille qui ont procédé à l'enterrement), tandis que les textes en arabe ont un caractère poétique (sur le plan "administratif», ils se contentent généralement de faire référence à l'année du décès). Afin d'illustrer cette différence fondamentale, j'ai reproduit les textes en latin et en italien. Les textes arabes sont d'ailleurs représentés en deux parties séparées ( $s a d r$ et ‘ajûz), selon la formule typique de la poésie arabe classique. Cette forme correspond à un discours structuré et organisé, typique de la poésie, avec un système binaire de cadences. C'est à ce niveau que s'établit, en arabe, la distinction entre la poésie (nadham) et la prose (nathr), la seconde étant caractérisée par un discours sans structure, forme et rythme codifiés.

A la suite de la traduction, je présente mes commentaires sur les versets que je considère les plus intéressants, surtout vis-à-vis de la problématique du lieu, de la "place» (au sens propre et figuré) et donc de la relation entre le lieu d'origine et la nouvelle vie vécue à Livourne.

1. Na`amat-Allah Cubbe

\author{
HIC IACET \\ GRATIA DE GHANTVZ CVBBE \\ PATRICIVS MARONITA HIERAPOLITANVS \\ VIR PIETATIS IN DEVM SINGVLARIS INTEGRITATIS INCORRVPTAE \\ MISERATONE IN AERVMNOSOS AFFECTV IN SVOS COMMEMORABILIS] \\ REI MERCATORIAE PERITISSIMVS DE PATRIA ET AMICIS \\ PRVDENTIA OPERA CONSILIIS OPTIME MERITVS \\ OBIIT DIE V OCTOBRIS MDCCCXXVIII \\ MICHAEL ALOYSIVS RAPHAEL FRATRI CARISSIMO MOERENTES
}

H M P

II. Pour réaliser ces traductions, j’ai bénéficié de l'aide précieuse de deux spécialistes français que je tiens à remercier chaleureusement; il s'agit de Madame Hager Boutarfa, enseignante d'arabe au lycée français de la Marsa (Tunis), et de Monsieur Adil Bennani, professeur agrégé d'arabe à Strasbourg. Grâce à eux, j’ai beaucoup appris sur la langue arabe et certaines de ses subtilités. Il est évident, toutefois, que j'assume la pleine responsabilité des textes publiés. 
Traduction du texte arabe

1. Que le Seigneur baigne par la pluie cette tombe où repose NàamatAllah, dont la disparition nous a ravagé le cœur.

2. Fils de nobles descendants de nobles, il a suivi le chemin parcouru par ses glorieux et augustes ancêtres,

3. Il a pratiqué la religion de Dieu comme un ascète, avec une foi qui ne lui a jamais fait défaut,

4. Sa main n'a jamais rien refusé aux besogneux, et ceux qui attendaient son aide n'ont jamais été déçus,

5. Il s'est démarqué par ses bienfaits et son intelligence fertile, ainsi que par la justesse de son jugement.

6. Il nous a laissé dans l'année du Christ 1828.

Le premier verset fait appel à l'image positive de la pluie qui baigne la terre, selon une vision poétique liée au symbolisme de la fertilité, typique d'une région orientale où l'eau est rare et bienfaisante. On retrouve cette "culture de l'eau" chez tous les Arabes (il suffit de penser, à titre d'exemple, aux fontaines des palais musulmans d'Andalousie). Le texte arabe fait d'ailleurs littéralement référence au "sable» où le corps est enterré, ce qui contribue à insérer encore davantage l'image dans le contexte d'un paysage assoiffé d'eau, aride, brulé par le soleil, presque désertique.

Le deuxième verset marque l'attachement à la patrie d'origine dans une logique de continuité: le défunt a continué, à Livourne, à parcourir la voie des ancêtres par une vie inspirée des valeurs religieuses. En d'autres termes, le texte s'adresse aux Arabes catholiques d'Alep qui viennent au sanctuaire de Montenero; il vise à leur montrer l'attachement du disparu à ses racines et, en même temps, sa capacité à reproduire, même loin de sa patrie, les comportements vertueux des ancêtres syriens.

2. Michel (Mikhaïl) Cubbe

\author{
HIC JACET \\ MICHAEL DE GHANTVZ CVBBE \\ PATRICIVS MARONITA HYERAPOLITANVS \\ QVI \\ PIETATIS OFFICIA HONESTATIS ET BENEFICENTIAE \\ EXEMPLO MAIORVM ADAMAVIT EXPLEVIT \\ SVIS IVCVNDISSIMVS ET AMICIS NEMINI VNQVAM INFENSVS \\ DECESS: IN PACE XPI: IV: NON : OCTOBR: AN : MDCCCXXX \\ ALOYSIVS ET RAPHAEL FRATRI CONCORDISSIMO \\ $\mathrm{M}$ : CVM LACRIMIS POSVERVNT
}


Traduction du texte arabe

1. Avez-vous, frères, des yeux capables de pleurer, avec la douleur et les larmes suffisantes,

2. Celui dont la mort nous a brûlé les entrailles, descendant d'une famille et d'une lignée illustres,

3. Vertueux, défenseur de la religion, fidèle et juste, homme d'une piété éclatante, fervent dans la foi?

4. Les ventres des femmes désireuses d'enfanter un homme aussi généreux sont devenus stériles.

5. Satisfait de son âme, le Miséricordieux a irrigué la terre de sa tombe par d'abondantes pluies.

6. Et si quelqu'un vous demande: "Quel est son sort?», répondez-lui que Cubbe a obtenu la béatitude de l'Au-delà. 1830

Avec ce texte, on instaure un véritable dialogue entre celui qui lit l'épitaphe et le poète, qui explique au lecteur ce qu'il faudra dire à ceux qui demanderont, de retour à Alep, des nouvelle de Michel Cubbe. Cette dimension dialogique se retrouve, de manière plus accentuée encore, dans l'épitaphe de son frère Luîs (voir ci-dessous, $\mathrm{n}^{\circ} 4$ ).

Il y a également, comme dans le texte précédent, une référence claire aux origines familiales (verset 2) et au rôle positif de l'eau qui baigne la terre où il repose, comme une reconnaissance divine de ses vertus (car Dieu est satisfait de lui).

3. Jean (Hanna) D’Andrea

\author{
$\Omega \times P \Omega$ \\ QUI RIPOSA LA SALMA \\ DEL CAVALIERE GIOVANNI MARCHESE D'ANDREA \\ MARONITA \\ NATO IN ALEPPO NELL ANNO 1770 \\ DAL BAGNO TERMALE DI CASCIANA PRESSO IL MONTE PARLASCIO \\ OVE CERCANDO ALL' EGRO ${ }^{12}$ CORPO SALUTE \\ TROVO LA MORTE \\ IL 2J DI OTTOBRE DELL ANNO 1839 \\ NEL DECIMO QUARTO LUSTRO DELLA VITA \\ DALLA PIETA DELLA CONSORTE E DELLA FIGLIA \\ IN QUESTO SACRO LOCO TRASPORTATA
}

I2. Egro: malade, infirme. 


\author{
A CONFORTO DEL LORO DOLORE \\ A TESTIMONIANZA DEL LORO AFFETTO \\ MEMORIA SEMPITERNA DELLE VIRTU DI LUI \\ CHE FU MARITO FEDELE TENERO PADRE \\ ONESTO CITTADINO PERFETTO CRISTIANO \\ MERITANDO LE LACRIME DEI CONGIUNTI \\ IL COMPIANTO DEGLI AMICI \\ LE PREGHIERE DEI FEDELI
}

\title{
Traduction du texte arabe
}

1. Ô famille D'Andrea, arrête tes pleurs et cesse ton deuil pour celui qui nous a quitté dans les bains.

2. Le Seigneur l'a choisi parmi vous, car il a accompli l'effort suprême dans la voie de la religion [jihâd]

3. Dieu l'a récompensé en posant sur sa tête la couronne de la Gloire éternelle et l'a accueilli auprès de Son Trône parmi Ses serviteurs.

4. Ainsi, grâce à ses mérites, le Protecteur divin l'éleva vers Lui.

Année 1839

Au premier verset apparaît une référence immédiate, presque crue, au lieu de la mort: les bains thermaux de Casciana, près de Pise. Ces bains sont cités également dans le texte en italien, avec plus de précision. Il est intéressant, je crois, de remarquer que cette citation a provoqué les protestations de certains fidèles de Casciana qui venaient au sanctuaire chaque année en pèlerinage auprès de la Vierge de Montenero. En effet, ils considéraient que ce texte donnait une image négative de leur village à tous ceux qui le lisaient (la pierre tombale se trouve dans un endroit où il y a beaucoup de passage, et elle est bien visible). En 1988, le maire communiste de Casciana avait ainsi songé à demander que cette inscription tombale soit effacée ou cachée ${ }^{13}$.

13. En 1988, un article du quotidien italien La Nazione a été consacré à cette question. Cet article, paru dans une partie du quotidien concernant l'actualité locale, est assorti d'une grande photographie de la tombe de Jean D'Andrea, où l'on peut lire clairement toute l'inscription en italien. Le journaliste écrit, à propos de la phrase sur la mort du marquis dans les bains thermaux de Casciana, que «des milliers de personnes chaque année, et depuis longtemps, lisent cette phrase qui sonne comme un reproche post mortem aux eaux thermales de Casciana». Et rajoute ensuite qu'à Casciana "quelqu'un", y compris le maire communiste du village, "commence à se demander s'il n'est pas opportun de faire enlever la pierre tombale ou de trouver un moyen 
Au deuxième verset nous trouvons le terme arabe jihâd, souvent (mal) traduit par "guerre sainte». J'ai préféré le traduire en indiquant la signification principale du terme arabe, valable pour les musulmans également, à savoir «l'effort suprême dans la voie de la religion". En d'autres termes, il s'agit d'un effort spirituel, qui n'a rien à voir avec la violence physique que l'on associe malheureusement trop souvent à ce terme, surtout en parlant de l'Islam.

Dans le troisième et le quatrième verset, il est fait référence au lieu ultime, au séjour du défunt après la mort, vu comme l'aboutissement d'un vie terrestre durant laquelle Jean d'Andrea a été partagé entre deux lieux: Alep et Livourne.

pour effacer la référence au lieu où le titulaire de la même pierre tombale est décédé». Cette pierre tombale "n'est pas élogieuse pour Casciana», affirme ensuite le curé du village. Le journaliste demande donc au curé s'il serait possible de la faire enlever, et la réponse est "nous pourrons essayer, mais ce ne sera pas facile». Selon le journaliste, cette pierre tombale "gâche la fête (spirituelle)" des fidèles de Casciana qui, chaque année, se rendent au sanctuaire de Montenero. Et le maire conclut qu'il cherchera à faire des démarches officielles (auprès des autorités locales de Livourne) pour « faire enlever la partie de l'inscription qui n'est surement pas une bonne publicité» pour son village. Dans l'article pourtant, le curé raconte que la famille D'Andrea était très attachée ("si affezionò ") à Casciana, où elle avait acheté un hôtel (devenu ensuite le siège d'une banque) et où elle a même fait bâtir une chapelle familiale dans le cimetière local (chapelle où se trouvait, entre autres, la dépouille de Jean D'Andrea avant qu'elle soit transférée à Montenero). Dans l'article, il n'est pas fait référence à l'inscription en arabe, mais on souligne les particularités "orientales» du marquis D'Andrea et de sa famille. En effet, la phrase contestée est définie comme "pas très chrétienne» par le journaliste, et le curé mentionne l'origine syrienne du défunt; de plus, avec des erreurs flagrantes d'italien, Jean D'Andrea est défini comme étant "di religione cristiano-sirio-maronica [sic] ». A ce propos, il faut rappeler qu'il ne s'agit pas d'une religion différente de celle du curé interrogé: les Maronites sont en effet, eux aussi, catholiques, et par rapport aux «Latins» ils ont simplement une différence de rite (dont la définition correcte est «siromaronita»). En conclusion, on a l'impression qu'on souligne les prétendues différences ethniques et «religieuses» du marquis D’Andrea pour mieux corroborer la volonté de faire enlever ou cacher la pierre tombale. Une volonté commune au maire communiste et au curé du village, comme dans certains épisodes pathétiques des films de Peppone et Don Camillo, vieilles images grotesques d'une "guerre froide" provinciale et campagnarde. Toutefois, après la lecture de cet article, si l'on pense à l'importance de la famille D'Andrea dans l'histoire de la région, le constat est plus sombre. On ne peut en effet éprouver que de la tristesse face à ces tentatives de mutiler les vestiges de sa présence et de son rôle prestigieux au sein du monde catholique. Heureusement, la pierre tombale se trouve encore intacte à côté de l'entrée principale du sanctuaire. P. C., "Che cattiva pubblicità da quell'antica lapide!» (ma traduction de l'italien pour les citations). 
4. Louis (Luîs) Cubbe

A XP $\Omega$
ALOISIO ANTONII F DE GHANTVZ CVBBE
PATRICIO HIERAPOLITANO PRISCAE NOBILITATIS
CONVENAE ET CIVI LIBVRNENSI
INGENVO INTEGRO PIENTISSIMO
QUEM OB FIDEM ET LENITATEM IN OMNES
OPTIMVS QVISQVE COMMENDAVIT
ET HEV NIMIVM PRAEPROPERE
ANTISTITI FRATRI TVMVLO PAREM
TRISTI LVCTV DEFLEVIT
IOANNES HAERES EX TESTAMENTO
PATRVELI SVAVISSIMO B M
HVNC TITVLVM AMORIS POSVIT
PLACIDE DECESSIT PROPE OCTOGENARIVS
XVII KAL IANVAR AN M DCCC XXXXII
VALE SALVE ALOISI
ALOISI NVMQVAM MEMORIATE SATIS
VALE SALVE
CITO TE XPTVS IN PACE

Traduction du texte arabe

1. Ô, toi qui lis, apporte mes salutations à ma famille, de laquelle j’ai été séparé par la distance,

2. Loue la ville d'Alep [ash-shahabâ] dont je suis le fils; jamais je n'ai cessé mon alliance avec ma famille qui habite cette ville.

3. Si quelqu'un te demande "Louis Cubbe, où est-il?», tu répondras «Hélas! Il a été ravi par la mort!»;

4. Mais toi, pour l'amour du Ciel, n'annonce pas d'une telle manière la douloureuse nouvelle, et dis seulement, à tous, «Louis vous a quittés, 5. Et quand la mort s'est annoncé, il a dit "Sachez-le, je vous ai quittés dans l'espoir de vous retrouver" ".

Année 1842.

Dans les versets 1 et 2 apparaît, une fois encore, l'attachement aux origines (famille et ville natale). D'ailleurs, la ville d'Alep est désignée par le terme ash-shahabầ (qui signifie, littéralement, «le nourrisson de la chamelle", au lieu du nom officiel, Halab), connu seulement par les Aleppins et par ceux qui connaissent bien sa région; cela confirme que le poète vise les chrétiens d'Alep comme destinataires privilégiés de son œuvre. On remarque également la valeur objective, non péjorative, de la distance entre Alep et Livourne, présentée comme une fatalité. On 
pourrait en conclure que si, dans la vie terrestre, la situation est importante pour exprimer les origines et comme repère identitaire (Alep), les qualités humaines (morales, religieuses), quant à elles, n'ont pas de barrières dans l'espace, et s'expriment à Livourne comme ailleurs.

Au verset 3, on observe, comme dans le poème sur la tombe de Michel Cubbe, que le poète instaure un véritable dialogue avec le lecteur, donc avec ceux qui visitent la tombe de ce dernier. Avec l'expression «où estil?», on souligne qu'on parle de quelqu'un qui est parti, qui a quitté son lieu d'origine.

Puis, dans les versets 4 et 5 , le poète suggère un parallèle entre le départ de sa ville natale et la mort. L'Au-delà devient le lieu de rencontre final, qui dépasse la séparation avec la famille (séparation vécue dans la vie sur terre). Une manière nette de «retrouver sa place» pour l'éternité.

Guido Bellatti Ceccoli

Université de Fribourg 


\section{BIBLIOGRAPHIE}

Anaissi, Tûbiya, Collectio Documentorum Maronitarum, Livorno, Fabbreschi, 1921.

Bellatti Ceccoli, Guido, "Da Kûbbâ a Livorno. Vita di monsignor Raffaele De Ghantuz Cubbe», in Dall'amicizia al dialogo. Saggi in onore di mons. Alberto Ablondi, a cura di Valdo Bertalot, Giampiero Bof, Riccardo Burigana, Adriano Fabris, Roma, Società Biblica Britannica \& Forestiera, 2004, p. 403-424.

—, «Medioriente livornese. La presenza di cattolici arabofoni a Livorno nei secoli XIX-XX», in Storia della diocesi di Livorno (1806-2006), a cura di Riccardo Burigana, Chiara Barovero, Livorno, Editasca, 2007, vol. II, p. 1-22.

-, Tra Toscana e Medioriente. La storia degli arabi cattolici a Livorno (sec. XVII-XIX), Livorno, Editasca, 2008.

—, "Voci dall'Oriente. Arabi cristiani e musulmani a Livorno in età moderna", in Livorno 1606-1806. Luogo di incontro tra popoli e culture, a cura di Adriano Prosperi, Torino/London/Venezia/ New York, Umberto Allemandi \& C., 2010, p. 418-429.

Bellatti Ceccoli, Guido, Di Nicola, Giordano, "Lapidi arabe a Livorno. Testimonianze cristiano-maronite del XIX secolo", Oecumenica Civitas, Quaderno II (2002), p. 85-96.

—, «Segni orientali nella Livorno ottocentesca. Epitaffi arabi nei luoghi di culto cristiani», I Tre Anelli - Les Trois Anneaux, 7 (2004), p. 83-115.

Litrico, Lorenza, «Un cedro del Libano in Toscana. Percorsi di ricerca sulla presenza dei maroniti a Livorno", Oecumenica civitas, Quaderno II (2002), p. 71-83.

Moosa, Matti, The Maronites in the History, New York, Gorgias Press, 2005.

Notain Darauni, Giovanni, Cenni storici sulla nazione siro-maronita, Livorno, Tipografia F. Vigo, I89o.

P. C., "Che cattiva pubblicità da quell'antica lapide!», La Nazione, vendredi 26 février 1988. 
